\title{
La influencia de los estilos de aprendizaje en el rendimiento académico de los estudiantes de la EAP de Tecnología Médica de la Facultad de Medicina de la UNMSM, Lima, Perú 2011
}

\author{
Giuliana Romero, Luis Marocho, Luis Solano, Elizabeth Pareja, Esther Valencia
}

Instituto de Medicina Tropical Daniel A. Carrión, Facultad de Medicina, UNMSM

Objetivos: Establecer, identificar y analizar la relación que existe entre los estilos de aprendizaje en el rendimiento académico de los estudiantes de Laboratorio Clínico y Anatomía Patológica de la Escuela Académico Profesional de Tecnología Médica de la UNMSM.

Diseño: Observacional, exploratorio, transversal, retrospectivo.

Institución: Instituto de Medicina Tropical Daniel A. Carrión, Facultad de Medicina, UNMSM.

Participantes: Alumnos de la EAP de Tecnología Médica de la Facultad de Medicina de la UNMSM.

Intervenciones: Análisis estadístico con el programa SPSS.

Principales medidas de resultados: Estilos de aprendizaje y rendimiento académico de los estudiantes.

Resultados: El estilo reflexivo alcanzó los mayores puntajes, seguido del estilo teórico. Existió correlación de $90 \%$ en la relación entre los estilos de aprendizaje y el rendimiento académico de los estudiantes de Laboratorio Clínico.

Conclusiones: Se determinó el rol del profesor en el modelo pedagógico centrado en el aprendizaje.

Palabras clave: Estilos de aprendizaje, rendimiento académico, cuestionario Honey Alonso.

\section{Himen complaciente en adolescentes y evaluación médico-legal de integridad sexual. División Médico Legal Lima Norte 2012}

Roger Velásquez, Denisse Astuhuamán, Max León

División Médico Legal Lima Norte, Instituto Medicina Legal, Ministerio Público

Introducción: Himen complaciente $(\mathrm{HC})$ o dilatable es aquel que se distiende sin dañarse durante la penetración en el acto sexual. Su hallazgo durante la evaluación médico-legal impide afirmar la existencia de penetración en el hecho investigado.

Objetivos: Determinar la prevalencia de $\mathrm{HC}$ en mujeres adolescentes evaluadas como presuntas víctimas de delito contra la libertad sexual (DCLS).

Diseño: Descriptivo, retrospectivo, transversal.

Institución: División Médico Legal Lima Norte, Instituto Medicina Legal, Ministerio Público.

Material de estudio: Certificados médico legales DCLS.

Intervenciones: Análisis de todos los casos de DCLS entre junio y julio 2012.

Principales medidas de resultados: Frecuencias, porcentajes.

Resultados: 133 adolescentes fueron evaluadas por presunto DCLS. La edad promedio fue 14,4 años. El agresor era conocido por la víctima en $73,7 \%$. En $10,5 \%$ un familiar estaba involucrado; $22,6 \%, 3 \%$ y $12 \%$ presentaron lesiones extragenitales, paragenitales y genitales, respectivamente. En $46,3 \%$ de casos se constató himen complaciente. En $3,8 \%$ y $9,8 \%$ se encontró desfloración reciente y signos de acto contranatura reciente, respectivamente. Presencia de espermios en fluidos en $8,3 \%$.

Conclusiones: El hallazgo de $\mathrm{HC}$ en adolescentes $(46,3 \%)$ es superior a la prevalencia general (36,9\%). A diferencia de otras series, en solo $1 / 5$ el agresor fue un familiar.

Palabras clave: Himen complaciente, adolescente, integridad sexual. 